\title{
Resistência à fratura de restaurações diretas com cobertura de cúspide em pré-molares superiores endodonticamente tratados
}

\section{Resistance to fracture of direct restorations with cuspal coverage in upper endodontically treated bicuspids}

\author{
Cássia Utiyama TAKAHASHI* \\ Antonio Alberto DE CARA** \\ Ivo CONTIN***
}

\begin{abstract}
TAKAHASHI, C. U.; DE CARA, A. A.; CONTIN, I. Resistência à fratura de restaurações diretas com cobertura de cúspide em pré-molares superiores endodonticamente tratados. Pesqui Odontol Bras, v. 15, n. 3, p. 247-251, jul./set. 2001.
\end{abstract}

\begin{abstract}
Dentes tratados endodonticamente são considerados mais susceptiveis à fratura em função da perda de estrutura dentária. Este estudo teve como objetivo avaliar o aumento da resistência à fratura de pré-molares superiores que receberam acesso endodôntico e foram restaurados em resina composta com cobertura de cúspide. Foram utilizados 40 pré-molares superiores humanos extraídos, que foram divididos em 4 grupos: I - dentes hígidos; II - dentes com acesso endodôntico, com preparo MOD, restaurados em resina composta, sem cobertura de cúspide; III - dentes com acesso endodôntico, com preparo MOD e redução de cúspide, restaurados em resina composta, com cobertura de cúspide; IV - dentes com acesso endodôntico, com preparo MOD, sem receber restauração. Os corpos-de-prova foram submetidos a teste de compressão até a fratura e os resultados foram submetidos a análise de variância e comparados através do teste de Tukey, podendo-se concluir que os dentes do grupo III (restaurados com cobertura de cúspide) apresentaram maior resistência à fratura, com valores significativos, em relação aos dentes do grupo II (restaurados sem cobertura de cúspide) e IV (só preparo cavitário sem restauração). A restauração em resina composta direta com cobertura de cúspide pode ser considerada uma alternativa restauradora para dentes pré-molares submetidos à terapia endodôntica.
\end{abstract}

UNITERMOS: Cúspide; Fraturas dos dentes; Tratamento do canal radicular; Resinas compostas.

\section{INTRODUÇÃO}

A escolha da melhor solução restauradora para dentes posteriores tratados endodonticamente, em muitos casos, não é uma questão de fácil resolução para o cirurgião-dentista. Os dentes despolpados estão mais susceptiveis à fratura em relação ao dente sadio ${ }^{1,2,4,9}$, tanto por fatores mecânicos (a perda de estrutura, rompimento do teto da câmara pulpar) como por fatores biológicos (alteração nas propriedades fisicas da dentina). Segundo HOWE; McKENDRY $^{6}$ (1990), dentes com preparos MOD e acesso endodôntico têm a resistência à fratura reduzida a 55\% em relação a dentes que apresentam apenas o preparo cavitário tipo MOD.

A restauração de dente tratado endodonticamente deve seguir uma série de princípios, devendo proporcionar resistência adequada a forças horizontais e axiais, bem como restabelecer forma e função. As restaurações sem cobertura de cúspide, quando submetidas a cargas axiais, exercem verdadeiro efeito de cunha, levando a uma deflexão das cúspides. Isto torna-se mais crítico em um dente posterior despolpado, que pode ter a profundidade da cavidade de 3 a 4 vezes maior do que um dente vitalizado, aumentando a deflexão das cúspides quando submetidas a cargas e conseqüentemente sofrendo maior risco de fratura ${ }^{4}$. Segundo VALE $^{18}$ (1959), e LARSON et al. ${ }^{7}$ (1981), se o istmo da cavidade é maior que um terço da distância entre as pontas de cúspide, deve ser realizada uma cobertura total ou parcial das cúspides.

As restaurações protéticas com proteção de cúspide (incrustações metálicas fundidas, "onlays" de porcelana, coroas metalocerâmicas) são consideradas a melhor indicação para dentes posteriores tratados endodonticamente ${ }^{5,8,12,15}$, promo-

*Mestranda do Departamento de Prótese; **Professor Doutor do Departamento de Dentística; ***Professor Doutor do Departamento de Prótese - Faculdade de Odontologia da USP. 
TAKAHASHI, C. U.; DE CARA, A. A.; CONTIN, I. Resistência à fratura de restaurações diretas com cobertura de cúspide em pré-molares superiores endodonticamente tratados. Pesqui Odontol Bras, v. 15, n. 3, p. 247-251, jul./set. 2001.

vendo a proteção do remanescente dentário, bem como o restabelecimento de sua forma e função, uma vez que há uma redistribuição de cargas, minimizando o efeito de cunha.

Porém, existem alguns casos em que as restaurações protéticas não podem ser utilizadas, como por exemplo, em função de prognóstico duvidoso de tratamento endodôntico (as restaurações protéticas geralmente não são realizadas enquanto a reparação periapical não puder ser confirmada radiograficamente $)^{4}$, situação de saúde geral comprometida, alto nível de cárie, situação periodontal, disponibilidade de tempo ou mesmo por fatores econômicos. Além disso, em dentes pré-molares superiores, não é raro ocorrerem lesões cariosas que levam facilmente ao comprometimento pulpar, sem que no entanto haja grandes destruições da estrutura dentária, principalmente quando acometidos por cáries proximais. Nestes casos, a tendência do cirurgião-dentista é optar por restaurações plásticas convencionais, deixando o elemento dentário bastante susceptivel à fratura.

A restauração em resina composta, com cobertura de cúspide seria uma opção para estes casos. Esta solução restauradora, embora utilizada rotineiramente por alguns profissionais, não apresenta referências na literatura.

Este trabalho teve como objetivo avaliar a resistência à fratura de dentes tratados endodonticamente, restaurados em resina composta, com cobertura de cúspide.

\section{MATERIAIS E MÉTODO}

Foram utilizados 40 pré-molares superiores, extraídos por indicação ortodôntica, mantidos hidratados em solução de cloreto de sódio $0,9 \%$ até o final do experimento.

Os espécimes tiveram a porção radicular incluída em tubos de PVC de $1 / 2$ polegada, com resina acrílica quimicamente ativada, até $1 \mathrm{~mm}$ do limite amelocementário, simulando a situação do nivel ósseo de sustentação do dente periodontalmente sadio, havendo o cuidado de inclui-los com o longo eixo do dente o mais perpendicularmente possivel à base do tubo de PVC, permitindo maior uniformidade de contato de cada espécime com o dispositivo de aplicação de força da máquina de teste de compressão.

Foram então classificados por ordem de tamanho e distribuídos em 4 grupos, de tal forma que houvesse a mesma variedade de forma e volume. Os espécimes foram divididos em 4 grupos de teste: grupo I - dentes hígidos, sem restaurações prévias ou evidências de cáries; grupo II - dentes que receberam preparo MOD, com exposição da câmara pulpar, restaurados em resina composta; grupo III - dentes que receberam preparo MOD com exposição da câmara pulpar e redução oclusal, restaurados em resina composta; grupo IV dentes que receberam preparo MOD com exposição da câmara pulpar e não foram submetidos a nenhum processo restaurador (Figura 1).

\section{Preparo}

Preparos MOD foram realizados nos espécimes dos grupos II, III e IV, utilizando-se pontas diamantadas cilindricas (KG Sorensen 2094), através de caneta de alta rotação refrigerada, fixada em uma base de microscópio óptico modificada, que possibilitava obter desgastes o mais paralelo possivel ao longo eixo do dente (Figura 2).

Este desgaste foi realizado com a profundidade de $4 \mathrm{~mm}$ a partir da crista mesial de cada dente. Foi realizada então uma exposição completa da câmara pulpar.

Os dentes do grupo III receberam também um desgaste plano da superficie oclusal de $1 \mathrm{~mm}$ a partir da crista distal do dente, sendo o desgaste maior nas cúspides e na crista mesial.

\section{Restauração}

Os dentes dos grupos II, III e IV tiveram a câmara pulpar selada por guta-percha em bastão aquecida e cimento de fosfato de zinco.

Os dentes do grupo II foram então restaurados com sistema adesivo Prime Bond 21 e resina composta (Herculite XRV), através de técnica incremental, seguindo as recomendações do fabricante (Figura 1 ).

Os dentes do grupo III foram restaurados com os mesmos materiais do grupo II procurando devolver a anatomia oclusal original, tendo o cuidado de obter no mínimo $1 \mathrm{~mm}$ de espessura de resina em toda a superficie oclusal (Figura 1). Os espécimes foram armazenados em solução de cloreto de sódio $0,9 \%$ por no mínimo 72 horas.

\section{Teste}

Todos os corpos-de-prova foram fraturados por força compressiva em máquina de ensaio universal RIEHLE, modelo FS-5, a uma aplicação de força em velocidade constante de $0,381 \mathrm{~mm}$ por minuto, através de uma ponta cilindrica (Figura 3) que contatava simultaneamente as arestas vestibular e lingual do corpo-de-prova (Figura 4).

Nos dentes do grupo II, houve o cuidado de não aplicar a força sobre o material restaurador, mas sobre a estrutura dentária remanescente. 
TAKAHASHI, C. U.; DE CARA, A. A.; CONTIN, I. Resistência à fratura de restaurações diretas com cobertura de cúspide em pré-molares superiores endodonticamente tratados. Pesqui Odontol Bras, v. 15, n. 3, p. 247-251, jul./set. 2001.
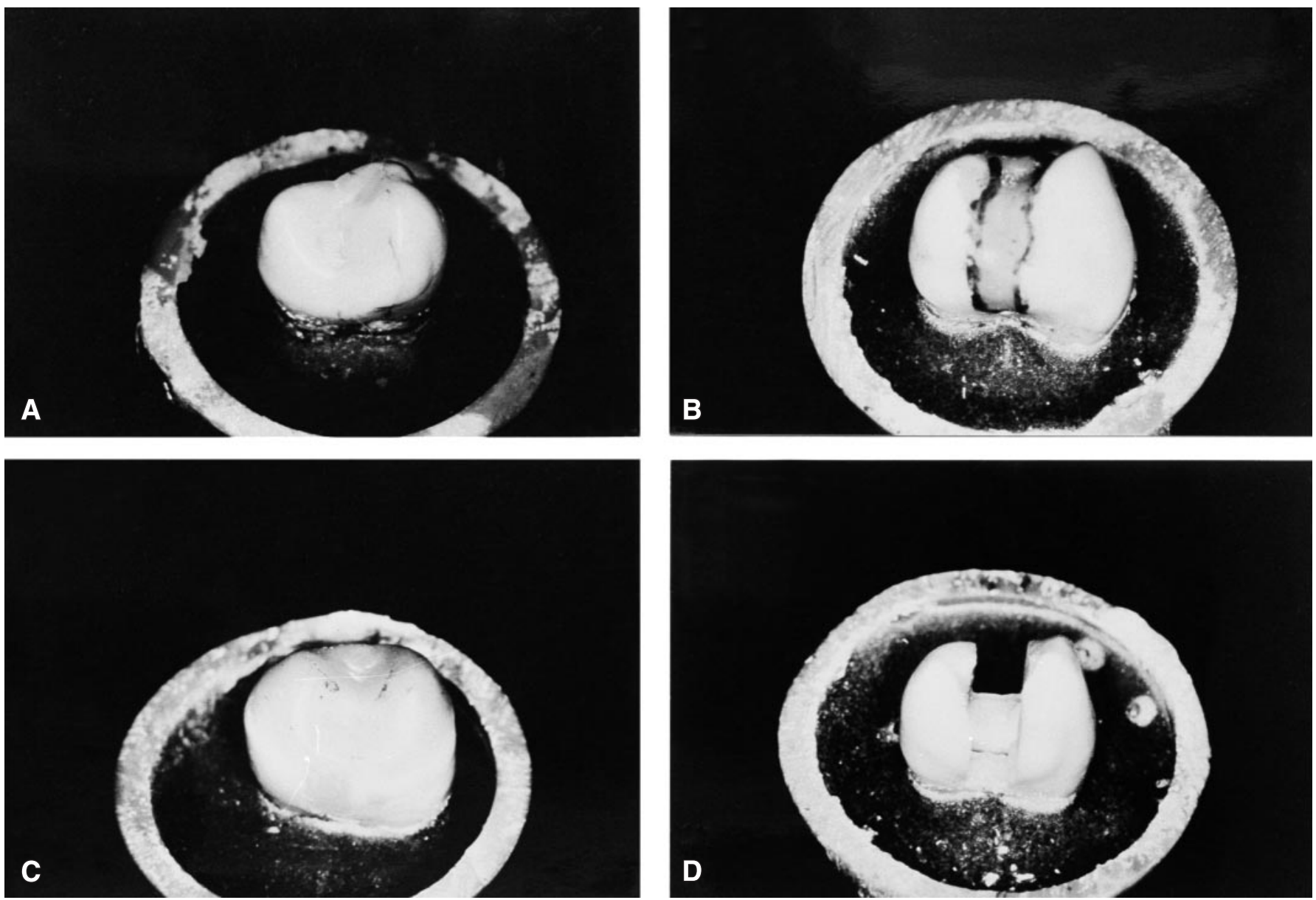

FIGURA 1 - Corpos-de-prova dos grupos I (A), II (B), III (C) e IV (D).

\section{RESULTADOS}

Os dados obtidos foram submetidos a análise de variância, sendo possivel observar através da tabela que existia ao menos uma diferença estatisticamente significante entre os 4 grupos estudados $(\mathrm{F}=20,41 ; \mathrm{p}=0,000)$. Para a comparação das médias foi utilizado o teste de Tukey (Tabela 1), indicando que houve diferença estatisticamente significante, com probabilidade de erro de $1 \%$ entre os grupos I e IV (hígidos e preparados), II e III (dentes restaurados sem cobertura de cúspide e com cobertura de cúspide), e III e IV (dentes restaurados com cobertura e dentes preparados). Não houve diferença estatisticamente significativa entre os grupos I e II e entre I e III. Com uma probabilidade de erro de $5 \%$, houve diferença significativa também entre os grupos II e IV.

\section{DISCUSSÃO}

Muitos trabalhos laboratoriais mostraram que preparos cavitários podem causar uma diminuição significativa na resistência à fratura dos elementos dentários ${ }^{12,13}$, em função do aumento da deflexão das cúspides frente à aplicação da carga mastigatória ${ }^{4}$. Em dentes posteriores despolpados, a deflexão é maior devido ao alargamento das cavidades (pela extensão da lesão cariosa, forma anatômica da polpa) bem como à maior profundidade da cavidade em função da terapia endodôntica ${ }^{4}$.

Os resultados de nosso estudo mostraram que a restauração de dentes pré-molares em resina composta, com cobertura de cúspide proporcionou um aumento significante da resistência à fratura, em relação aos dentes preparados ou restaurados sem cobertura de cúspide.

Tanto em estudos in vitro ${ }^{8,10,12}$ como acompanhamentos in vivo ${ }^{14}$ observou-se o aumento da resistência à fratura de dentes restaurados em amálgama com cobertura de cúspide, em relação a dentes com largas restaurações MOD de amálgama. HANSEN et al. $^{4}$ (1990) mostraram que restaurações definitivas ou temporárias em amálgama para dentes despolpados eram aceitáveis apenas quan- 
TAKAHASHI, C. U.; DE CARA, A. A.; CONTIN, I. Resistência à fratura de restaurações diretas com cobertura de cúspide em pré-molares superiores endodonticamente tratados. Pesqui Odontol Bras, v. 15, n. 3, p. 247-251, jul./set. 2001.

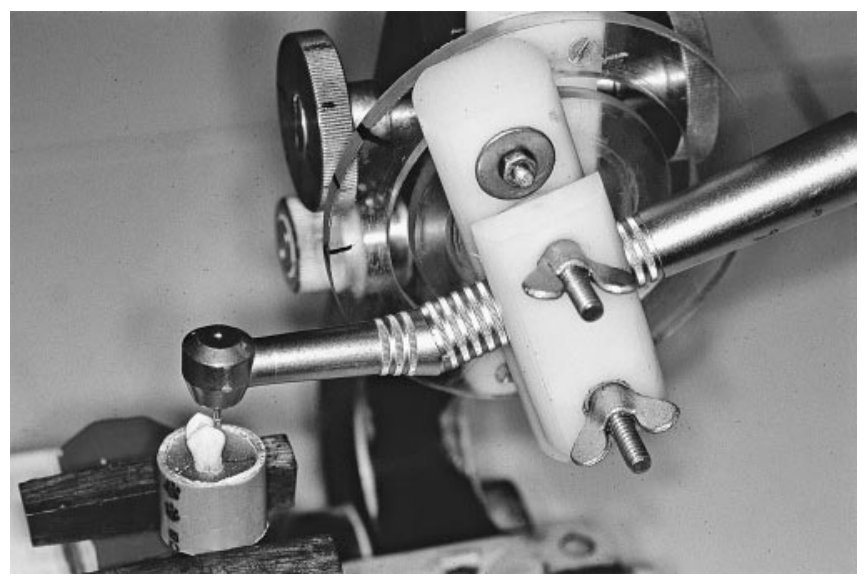

FIGURA 2 - Corpo-de-prova recebendo preparo MOD na máquina de preparos.

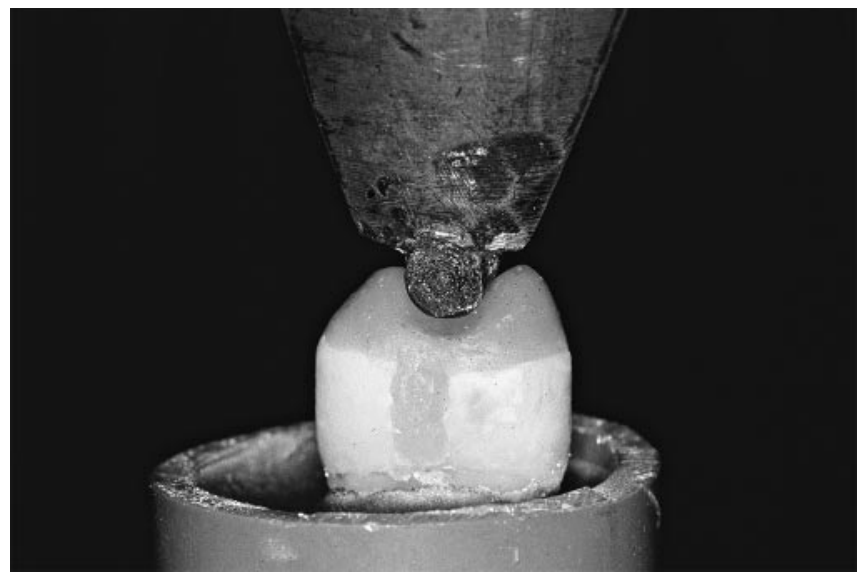

FIGURA 4 - Corpo-de-prova do grupo III sob teste de compressão.

do realizadas com cobertura de cúspide mínima de 2 a $3 \mathrm{~mm}$, e também propôs a restauração em resina composta como uma alternativa de tratamento. Segundo ULUSOY et al. ${ }^{17}$ (1991), restaurações em resina composta ou amálgama, com proteção da cúspide lingual seria uma alternativa para dentes pré-molares superiores endodonticamente tratados. ROBBINS ${ }^{11}$ (1990) e LINN; MESSER ${ }^{9}$ (1994) indicam a cobertura de cúspides para restaurar dentes posteriores despolpados, considerando inclusive a cobertura de cúspide mais importante do que a preservação de estrutura dentária.

Este melhor comportamento das restaurações com cobertura de cúspide poderia ser justificada pela melhor distribuição das cargas, neutralizando o efeito de cunha das restaurações MOD sobre o remanescente dentário, que levaria à deflexão das cúspides e possivel fratura ${ }^{5}$.

Contudo, é preciso ressaltar que as condições

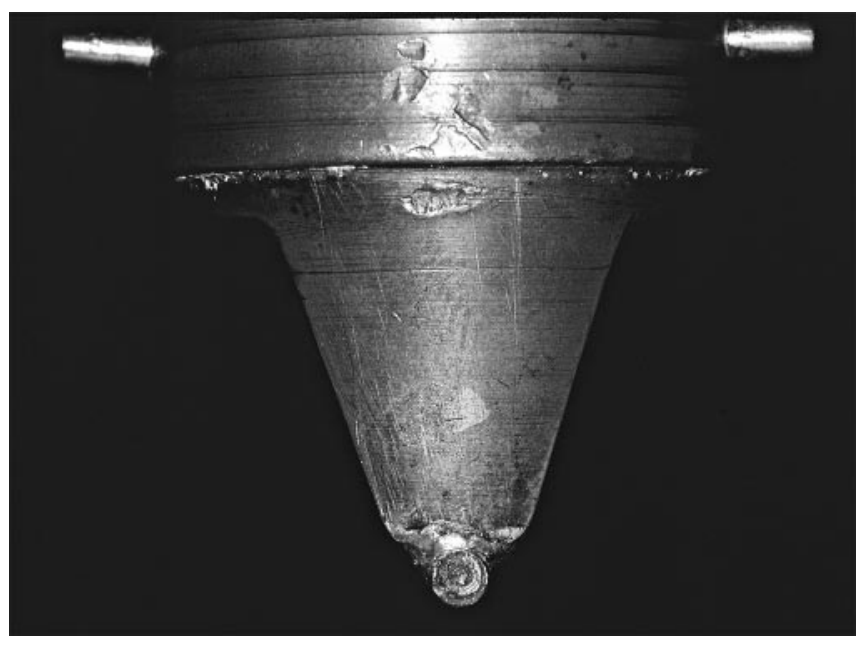

FIGURA 3 - Dispositivo utilizado para aplicar a força de compressão sobre os corpos-de-prova.

TABELA 1 - Médias de resistência à fratura (kgf) e comparações pelo teste de Tukey.

\begin{tabular}{|c|c|c|c|c|c|}
\hline \multicolumn{2}{|c|}{ Dentes } & \multirow{2}{*}{ Média } & \multirow{2}{*}{$\begin{array}{l}\text { Desvio- } \\
\text { padrão }\end{array}$} & \multirow{2}{*}{\begin{tabular}{|c|} 
Médias \\
compara- \\
das
\end{tabular}} & \multirow{2}{*}{$\begin{array}{l}\text { Significân- } \\
\text { cia }(\%)\end{array}$} \\
\hline Condição & Grupo & & & & \\
\hline Íntegro & I & 75,2 & 18,1 & I x II & $\mathrm{ns}$ \\
\hline $\begin{array}{c}\text { Sem } \\
\text { cobertura }\end{array}$ & II & 55,0 & 24,8 & I x III & ns \\
\hline $\begin{array}{c}\text { Com } \\
\text { cobertura }\end{array}$ & III & 91,7 & 22,0 & I x IV & $1 *$ \\
\hline \multirow[t]{3}{*}{$\begin{array}{c}\text { Sem } \\
\text { restauração }\end{array}$} & IV & 25,1 & 13,5 & II x III & $1^{*}$ \\
\hline & & & & II x IV & $5^{*}$ \\
\hline & & & & \begin{tabular}{|l|l} 
III x IV \\
\end{tabular} & $1 *$ \\
\hline
\end{tabular}

*Valor crítico de Tukey $(1 \%)=30,45$; valor crítico de Tukey $(5 \%)=24,36 ;$ ns $=$ não significante.

de aplicação de cargas utilizadas neste estudo são de natureza estática, quando comparadas à dinâmica das repetidas cargas às quais os dentes estão expostos na cavidade oral. Além disso, um fator crítico na avaliação dos resultados de testes com dentes extraídos é saber quais as alterações sofridas após a extração ${ }^{16}$. Devemos levar em conta a elasticidade dos tecidos orgânicos do dente, osso alveolar e ligamentos periodontais.

\section{CONCLUSÕES}

De acordo com a metodologia aplicada, pôde-se concluir que a restauração em resina composta com cobertura de cúspide de pré-molares extraí- 
TAKAHASHI, C. U.; DE CARA, A. A.; CONTIN, I. Resistência à fratura de restaurações diretas com cobertura de cúspide em pré-molares superiores endodonticamente tratados. Pesqui Odontol Bras, v. 15, n. 3, p. 247-251, jul./set. 2001.

dos, tratados endodonticamente, aumenta a resistência à fratura do remanescente dentário em rela- ção à restauração com o mesmo material sem recobrimento oclusal.

TAKAHASHI, C. U.; DE CARA, A. A.; CONTIN, I. Resistance to fracture of direct restorations with cuspal coverage in upper endodontically treated bicuspids. Pesqui Odontol Bras, v. 15, n. 3, p. 247-251, jul./set. 2001.

Endodontically treated teeth are considered more susceptible to fracture because of the loss of tooth structure. The aim of this study was to evaluate the increase of resistance to fracture of upper bicuspids that underwent endodontic access and were restored with composite resin, with cuspal coverage. Forty extracted human maxillary premolars were divided in 4 groups: I - intact teeth; II - teeth with endodontic access and MOD preparation, restored with composite resin, without cuspal coverage; III - teeth with endodontic access, MOD preparation and occlusal reduction, restored with composite resin, with cuspal coverage; IV - teeth with endodontic access and MOD preparation, without any restoration. The test specimens were submitted to compression test up to their fracture. The test of Tukey and the ANOVA analysis were used to compare and test the results. The teeth from group III (with cuspal coverage) presented with significantly greater resistance to fracture, when compared with those from groups II (restored without cuspal coverage) and IV (not restored). The composite restoration with cuspal coverage can be considered an alternative for endodontically treated premolars.

UNITERMS: Cusp; Tooth fractures; Root canal therapy; Composite resins.

\section{REFERÊNCIAS BIBLIOGRÁFICAS}

1. BLITZ, N.; SEROTA, K. S. Exploding the miths, defining the future. Oral Health, v. 85, n. 12, p. 19-24, Dec. 1985.

2. BURKE, F. J. T. Tooth fracture in vivo and in vitro. J Dent, v. 20, n. 3, p. 131-139, June 1992.

3. HANSEN, E. K. In vivo fracture of endodontically treated premolars restored with MOD amalgam or MOD resin fillings. Dent Mater, v. 4, n. 4, p. 169-173, Aug. 1988.

4. HANSEN, E. K; ASMUSSEN, E.; CHRISTIANSEN, N. C. In vivo fractures of endodontically treated posterior teeth restored with amalgam. Endod Dent Traumatol, v. 6, n. 2, p. 49-55, Apr. 1990.

5. HOOD, J. A. A. Biomechanics of the intact, prepared and restored tooth: some clinical implications. Int Dent $\mathbf{J}$, v. 41, n. 1, p. 25-32, Feb. 1991.

6. HOWE, J. C. A.; McKENDRY, D. J. Effect of endodontic access preparation on resistance to crown-root fracture. $\mathbf{J}$ Am Dent Assoc, v. 121, n. 6, p. 712-715, Dec. 1990.

7. LARSON, T. D.; DOUGLAS, W. H.; GEISTFELD, R. E. Effect of prepared cavities on the strength of teeth. Oper Dent, v. 6 , n. 1, p. 2-5, Winter 1981.

8. LIBERMAN, R.; JUDES, H.; COHEN, E. Restoration of posterior pulpless teeth: amalgam overlay versus cast gold onlay restoration. J Prosthet Dent, v. 57, n. 5, p. 540-543, May 1987.

9. LINN, J.; MESSER, H. H. Effect of restorative procedures on the strength of endodontically treated molars. $\mathbf{J}$ Endod, v. 20, n. 10, p. 479-489, Oct. 1994.

10. REAGAN, S. E; SCHWANDT, N. W.; DUNCANSON Jr., M.
G. Fracture resistance of wide-isthmus mesio-oclusodistal preparations with and without amalgam cuspal coverage. Quintessence Int, v. 20, n. 7, p. 469-472, July 1989.

11. ROBBINS, J. W. Guidelines for the restoration of endodontically treated teeth. J Am Dent Assoc, v. 120, n. 5, p. 558-562, May 1990.

12. SALIS, S. G.; HOOD, J. A. A.; KIRK, E. E. J; STOKES, A. N. S. Impact-fracture energy of human premolar teeth. $\mathbf{J}$ Prosthet Dent, v. 58, n. 1, p. 43-48, July 1987.

13. SALIS, S. G; HOOD, J. A. A.; STOKES, A. N. S.; KIRK, E. E. J. Patterns of indirect fracture in intact and restored human premolar teeth. Endod Dent Traumatol, v. 3, n. 1, p. 10-14, Feb. 1987.

14. SMALES, R. J. Longevity of cusp-covered amalgams: survivals after 15 years. Oper Dent, v. 16, n. 1, p. 17-20, Feb. 1991.

15. SORENSEN, J. A.; MARTINOFF, J. T. Intracoronal reinforcement and coronal coverage: a study of endodontically treated teeth. J Prosthet Dent, v. 51, n. 6, p. 780-784, June 1984.

16. STAMPALIA, L. L.; NICHOLLS, J. I.; BRUDVIK, J. S. Fracture resistance of teeth with resin-bonded restorations. J Prosthet Dent, v. 55, n. 6, p. 694-698, June 1986.

17. ULUSOY, N.; NAYYAR, A.; MORRIS, C. L. F.; FAIHURST, C. W. Fracture durability of restored functional cusps on maxillary nonvital premolar teeth. J Prosthet Dent, v. 66, n. 3, p. 330-335, Sept. 1991.

18. VALE, W. A. Cavity preparation and further thoughts on high speed. Br Dent J, v. 107, p. 333-346, Dec. 1959. 